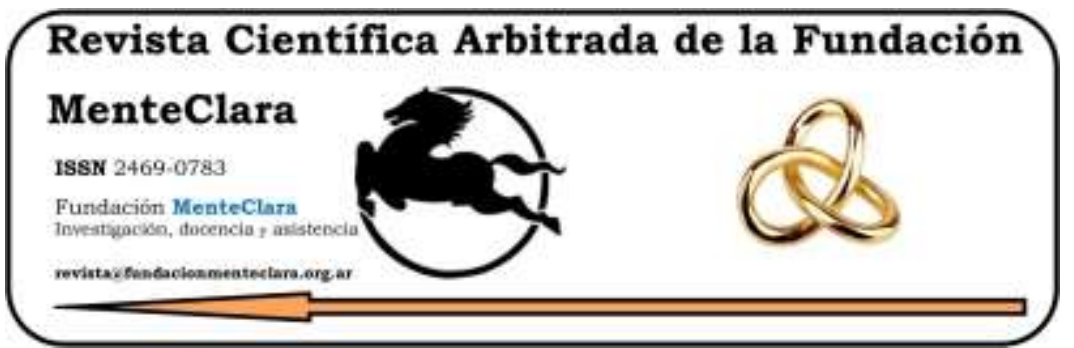

Artículos atravesados por (o cuestionando) la idea del sujeto -y su género- como una construcción psicobiológica de la cultura. Articles driven by (or questioning) the idea of the subject -and their gender- as a cultural psychobiological construction.

Vol. 5 (2020), enero-diciembre

ISSN 2469-0783

https: / / datahub.io/ dataset/2020-5-e149

\title{
LA ORIENTACIÓN A LA DOMINANCIA SOCIAL: UNA INVARIANTE PSICOLÓGICA EN LA LEGITIMACIÓN DE LAS FILOSOFÍAS DE DESIGUALDAD SOCIAL
}

\author{
ORIENTATION TO SOCIAL DOMINANCE: A PSYCHOLOGICAL INVARIANT IN THE \\ LEGITIMATION OF SOCIAL INEQUALITY PHILOSOPHIES
}

Luis Carlos Jaume ${ }^{1}$, Marcelo Agustín Roca², Susana Celeste Azzollini³

Cómo citar este artículo / Citation: Jaume L. C., Roca M. A. \& Azzollini S. C. (2020). "La Orie ntación a la Dominancia Social: una invariante psicológica en la legitimación de las filosofias de desigualdad social". Revista Científica Arbitrada de la Fundación MenteClara, Vol. 5 (149). DOI: https://doi.org/10.32351/rca.v5.149

Copyright: (C) 2020 RCAFMC. Este artículo de acceso abierto es distribuido bajo los términos de la licencia Creative Commons Attribution 4.0 International License (CC BY 4.0). Recibido: 31/03/2020. Aceptado: 01/04/2020 Publicación online: 02/04/2020

Conflicto de intereses: Ninguno que declarar.

\section{Resumen}

Distintos enfoques teóricos a lo largo de la historia han intentado explicar por qué las sociedades establecen ideologías que piensen las relaciones intergrupales como desiguales y jerárquicas, y por qué es aceptado por los individuos. Entre las perspectivas que ahondaron estas filosofias de la desigualdad se encuentran: el funcionalismo y el marxismo. Ambas visiones son incompletas y se centran en modelos top-down (pensando la dinámica de la sociedad de arriba hacia abajo). A la vez distintas teorías psicológicas, como la teoría de la identidad social, la teoría cultural ideológica, la teoría de la justificación del sistema y la teoría del conflicto

\footnotetext{
${ }^{1}$ Instituto de Investigaciones de la Facultad de Psicologia (UBA-CONICET) Universidad de Buenos Aires, Argentina.

2 Universidad de Buenos Aires, Argentina. marcelo87roca@gmail.com

3 Instituto de Investigaciones de la Facultad de Psicología (UBA-CONICET) Universidad de Buenos Aires, Argentina.
} 
realista, empezaron a investigar el factor psicológico en la adhesión a una ideología, virando los el foco hacia modelos bottom up. En los últimos años, la teoría de la dominancia social incorpora aportes de las teorias mencionadas y postula que existe un deseo de los individuos por instaurar y mantener jerarquías sociales, por medio de la subordinación de ciertos grupos a otros. Si bien este fenómeno se ha estudiado en distintos países de manera empírica, no se evidencian trabajos en español que hayan realizado una revisión teórica exhaustiva. Es por ello que el objetivo de este trabajo será realizar una revisión teórica sobre la SDT.

\section{Abstract}

Different theoretical approaches throughout history have tried to explain why societies establish ideologies that think intergroup relationships as unequal and hierarchical, and why it is accepted by individuals. Among the perspectives that deepened these philosophies of inequality are: functionalism and Marxism. Both visions are incomplete and focus on top-down models (thinking about the dynamics of society from top to bottom). At the same time different psychological theories, such as the theory of social identity, ideological cultural theory, the theory of system justification and the theory of realistic conflict, began to investigate the psychological factor in adherence to an ideology, turning the focus to bottom-up models. In recent years, the theory of social dominance, incorporates contributions from the aforementioned the aforementioned theories, and postulates that there is a desire of individuals to establish and maintain social hierarchies, through the subordination of certain groups to others. Although this phenomenon has been studied in different countries empirically, there is no evidence of works in Spanish that have carried out a comprehensive theoretical review. That is why the objective of this work will be to carry out a theoretical review on the SDT.

Palabras Claves: Teoría de la Dominancia Social; Orientación a la Dominancia Social; Ideología de la desigualdad

Keyw ords: Social Dominance The ory; Social Dominance orie ntation; Ideology of inequality 


\section{Introducción}

Distintos enfoques teóricos a lo largo de la historia han intentado explicar por qué las sociedades tienden a establecer ideologías que piensen las relaciones intergrupales como desiguales y jerárquicas, y porque sus individuos tienden a aceptarlo. Entre las perspectivas que ahondaron estas filosofias de la desigualdad se encuentran: el funcionalismo (Martín-Baró, 1983/2004) y el marxismo (Engels, 1884/1902) (Marx \& Engels, 1846/1970). Ambas asumen distintos roles al asignar una postura que es más neutral y otra que es más crítica a las ideologías partiendo de distintas concepciones sobre la sociedad y el hombre.

La primera piensa a la sociedad como un sistema unitario y coherente que se rige por un conjunto de valores y normas (Martín-Baró, 1983/2004). De esta manera, piensa que la ideología combina ideas y actitudes que de manera interdependiente se tienden a agrupar y que cumple una función positiva de organización funcional (Converse, 1964). La función de la ideología sería entonces organizar los representaciones sociales, posibilitar ciertas actitudes, controlar las creencias sociales y personales e influir sobre el comportamiento social, vehiculizando de esta manera la justificación del orden social existente y legitimándolo como universal.

Por otro lado, y desde la perspectiva marxista, se piensa a la sociedad en clases dominantes y proletariado. Así, los grupos dominados estarian alienados sin conocer la dominación a la que son expuestos, debido a que si tomaran conciencia de esta condición podrían rebelarse (Sidanius \& Pratto, 1999). En esta manera de pensar; la alienación, la conciencia de clase y la falsa conciencia, delimitan la comprensión en torno a porqué los individuos justifican la filosofia de la desigualdad que conforman. 
Las distintas teorías mencionadas han trabajado intentando abordar como ciertos grupos contribuyen a perpetuar una ideología de desigualdad social, económica y de género en cada sociedad. Sin embargo, ambas visiones son incompletas y se centran en modelos topdown - pensando la dinámica de la sociedad de arriba hacia abajo- (Jost, 2006). A su vez, distintas teorías psicológicas, como la teoría de la identidad social (Tajfel \& Turner, 1986), la teoría cultural ideológica (Adorno, Frenkel-Brunswik, Levinson \& Sanford, 1950), la teoría de la justificación del sistema (Jost, 2006) y la teoría del conflicto realista (Sherif, Harvey, White, Hood \& Sherif, 1961), empezaron a investigar el factor psicológico en la adhesión a una ideología, virando el foco hacia modelos bottom up (Jost, 2006). En los últimos años, la teoría de la dominancia social -en adelante SDT- (Pratto, Sidanius, Stallworth \& Malle, 1994), toma e incorpora aportes de las teoría mencionadas y generó una comprensión distinta de la dinámica de este tipo de conflicto intergrupal.

De acuerdo con la SDT existe un deseo por parte de los individuos por instaurar y mantener jerarquías sociales, por medio de la subordinación de ciertos grupos a otros.

Brewer (2001) postula que los individuos en toda sociedad tienden a organizarse en grupos en relación a un conjunto de valores, creencias o características compartidas, las cuales delimitan el grupo de pertenencia -endogrupo-y los otros grupos sociales -exogrupos-. A su vez, esto favorece una asimetría comportamental, determinada por la pertenencia grupal de los individuos (Ferguson \& Dyck, 2012).

Según esta teoría, la sociedad tiende a organizarse en sistemas basados en jerarquías, en donde uno de los grupos tiende a dominar por sobre los otros (Sidanius \& Pratto, 1999, 2004). De esta manera, el grupo 
dominante obtiene un valor social positivo desmesurado en comparación con los demás grupos ya sea a partir de un mayor poder político, influencia o estatus social, entre otros. Por lo que se tiende a minimizar o anular la conflictiva grupal, gracias a las ideologias que justifican la desigualdad social (Pratto, 2015).

Si bien este fenómeno se ha estudiado en distintos países de habla hispana de manera empírica (Cárdenas, Mesa, Lagues, \& Yañez, 2010) (Etchezahar, Prado-Gascó, Jaume, \& Brussino, 2014) no se evidencian trabajos en español que hayan realizado una revisión teórica exhaustiva. Es por ello que el objetivo de este trabajo es realizar una revisión teórica sobre la SDT.

\section{La Orientación a la Dominancia Social}

La orientación a la dominancia social suele definirse como la medida en que las personas desean y apoyan una jerarquía social a partir de la cual ciertos grupos "superiores" dominan sobre los "inferiores" y es considerada la base psicológica de la SDT (Sidanius \& Pratto, 1999, 2004). Esta jerarquía social se ve influenciada por la ideologia tanto de los grupos dominantes como por los subordinados, los mitos y su arraigo en distintos aspectos de la cultura, la creencia de una mayor objetividad y por último, el grado en el que un mito sirve de enlace entre el deseo de establecery mantener la jerarquía social, basada en los grupos, y el apoyo de las políticas sociales.

La SDT ha buscado dar cuenta de los factores que generan y mantienen estas jerarquías sociales. Sidanius y Pratto (1999) proponen el análisis de las estructuras jerárquicas a través de tres elementos constitutivos de la estructura jerárquica: la edad, el género y un conjunto de relaciones intergrupales arbitrarias (Pratto, Sidanius \& Levin, 2006) 
(Sidanius \& Pratto, 1999, 2004) (Sidanius, Pratto, van Laar \& Levin, 2004).

En relación a la edad y al género, diversos estudios transculturales muestran que los hombres suelen presentar una mayor orientación a la dominancia social que las mujeres, independientemente del contexto cultural en el cual se encuentren (Lee, Pratto \& Johnson, 2011) (Sidanius, Levin, Liu \& Pratto, 2000). Asimismo, los adultos tienen una mayor orientación a la dominancia social en relación a niños y jóvenes (Sidanius et al, 2004). Esto llevó a la concepción de la hipótesis de invarianza de edad y género (Cárdenas et al, 2010) (Jost \& Thompson, 2000) (Pratto et al, 2000) (Sidanius \& Pratto, 1999, 2004) (Silván-Ferrero \& Bustillos, 2007) (Zakrisson, 2008).

En cuanto al conjunto de relaciones intergrupales arbitrarias, el armado de jerarquías sociales a partir de distintas categorias permiten diferenciar y caracterizar a los grupos a partir de la raza, la etnia, la cultura, la religión, la nacionalidad, la orientación política o la clase social, entre otras (Pratto et al, 1994) (Pratto, Stallworth, Sidanius \& Siers, 1997) (Sidanius, Cotterill, Sheehy-Skeffington, Kteily \& Carvacho, 2017) (Sidanius et al, 2000) (Sidanius, Pratto \& Brief, 1995) (Sidanius \& Pratto, 1999) (Silván-Ferrero \& Bustillos, 2007).

Debido a estos tres elementos de la estructura de las jerarquías sociales, Sidanius y Pratto (2004) identifican tres aspectos centrales de la SDT. El primero de ellos refiere, como se señaló anteriormente, a que tanto la edad como el sexo existen de manera invariable en todo sistema social, mientras que existen otros sistemas jerarquizados que son arbitrarios y emergen producto de excedentes económicos. El segundo elemento da cuenta de que, en su mayoría, las formas de conflicto intergrupal y de opresión al exogrupo pueden ser comprendidas como 
una tendencia humana a formar jerarquías sociales basadas en grupos. En cuanto al último elemento, los autores sostienen que todo sistema social se encuentra sometido a una contraposición de fuerzas que tienden a debilitar o a reforzar la estructura jerárquica vigente.

La SDT identifica asimismo tres procesos que modulan esta estructura jerárquica (Sidanius \& Pratto, 1999, 2004), que se desarrollarán en los siguientes apartados: la discriminación individual, la discriminación institucional y la asimetría conductual.

\section{Discriminación individual}

Según la SDT la discriminación individual constituye un mecanismo que posibilita generary mantener la jerarquía social intergrupal, definida como un comportamiento negativo e injustificable hacia los miembros de un determinado grupo (Dovidio, Hewstone, Glick \& Esses, 2010) (Myers $\&$ Lamarche, 1992). La negativa de contratar a un empleado en virtud de su etnia, color de piel, nacionalidad, o género, suelen considerarse como ejemplos de discriminación individual (Bonanno \& Jost, 2006) (Jost, Glaser, Kruglanski \& Sulloway, 2003) (Lange, 1996).

Cuando existe una gran cantidad de actos individuales de discriminación a lo largo del tiempo, los mismos contribuyen a las diferencias de poder entre los grupos sociales. Es por ello que de acuerdo con la SDT la discriminación individual aporta a crear y/o reforzar las diferencias en términos de poder y riqueza entre grupos.

\section{Discriminación institucional}

La discriminación institucional refiere a los roles, procedimientos y acciones discriminatorios de las instituciones públicas o privadas 
llevadas a cabo ya sea de manera deliberada o inconscientemente (Sidanius et al, 2004). La discriminación institucional conlleva a una distribución desigual del valor social positivo y negativo en la jerarquía social (Feagin \& Feagin, 1978). En pos de mantener la integridad de la jerarquía social, las instituciones utilizan el terror sistemático, entendido como el uso de violencia o de amenazas de violencia desproporcionada dirigida contra los subordinados (Sidanius, 1993) (Sidanius \& Pratto, 1999). El terror sistemático contribuye a mantener relaciones expropiatorias entre los miembros del grupo dominante y los miembros del grupo subordinado, reforzando las diferencias entre ambos. De acuerdo con la SDT, es posible distinguir tres tipos de terror sistemático: el terror oficial, el terror semioficial y terror no oficial. El terror oficial se define como la violencia "legal", perpetrada por órganos del Estado y dirigida desproporcionadamente hacia miembros de grupos subordinados (Amnesty International USA \& United States of America, 1999) (Bernard 1993) (Tonry, 2010). El terror semioficial se define como el uso de la violencia contra las minorias por parte de las fuerzas de seguridad del Estado, tales como la policía o el ejército. A diferencia del terror institucional, en el terror semioficial no existe una sanción oficial del sistema político y judicial, sino que se trata de un comportamiento de las fuerzas que, aunque no se encuentra oficialmente legislado, es cultural e institucionalmente legitimado de manera extraoficial (Angus \& Crichlow, 2018). Por su parte, el terror no oficial se refiere a un acto perpetrado por individuos o grupos de individuos que pertenecen a los estratos más altos de la sociedad, que usan la violencia contra las minorías. Si bien este tipo de terror no tiene la aprobación activa de las agencias gubernamentales, por lo general tiene la aprobación y participación no activa de miembros de las fuerzas de seguridad (Alves, 
2019) (Commission Nationale Consultative des Droits de l'Homme [CNCDH], 1999) (Moore et al, 2018).

Siguiendo a Sidanius y Pratto (2004), resulta relevante destacar que los sistemas de justicia tanto legal como penal se encuentran entre los principales instrumentos utilizados para establecer y mantener la estructura jerárquica de las relaciones intergrupales. Para los autores, si bien la seguridad interna y los sistemas de justicia penal fueron diseñados para mantener el orden social, la ley ocasionalmente se escribe y se cumple en favor de los intereses de los grupos dominantes.

Cabe señalar que, de acuerdo con Sidanius y Pratto (2004), la discriminación contra los miembros de los grupos subordinados es observable en todas las sociedades con excedente económico, aún en aquellas de corte democrático e igualitario. No obstante, para el autor en las sociedades llamadas democráticas el nivel de brutalidad y discriminación contra los subordinados tenderá a ser algo limitado debido a la defensa de los ideales culturales de igualdad ante la ley. Es por ello que aunque el sistema de justicia penal continuará adoptando una manera discriminatoria, las élites dentro de estos sistemas se verán con algunos inconvenientes para justificar la presencia y el alcance de la discriminación.

\section{Asimetria conductual}

La construcción de asimetría conductual es una de las principales cualidades por las cuales se destaca la SDT en relación a otros modelos estructurales, tales como el marxismo, la teoría de la élite neoclásica y la teoría de la posición grupal (Sidanius \& Pratto, 1999). Mientras estos modelos mencionados postulan que los grupos dominantes hegemónicos oprimen, manipulan y controlan a los grupos subordinados, la SDT a la 
vez sugiere que los grupos dominados participan de manera activa en contribuir a su propia subordinación. La SDT no considera subordinados solamente a los que son objetos de opresión, sino también a individuos que suelen tener algo de participación activa en el ejercicio opresivo (Sidanius \& Pratto, 1999).

Por otro lado, la SDT reconoce que los subordinados resisten a su propia opresión y que en ocasiones esta resistencia puede ser intensa y conducir a rebeliones e incluso a revoluciones sociales. Sin embargo, la revolución social exitosa es un evento raro, y la gran mayoría de los sistemas de jerarquía social se mantienen relativamente estables por periodos prolongados de tiempo. De este modo si bien se reconoce que siempre existe un elemento de resistencia y resentimiento dentro de los grupos subordinados, se sugiere que en jerarquías usualmente estables de grupos, las actividades de los subordinados pueden puntuarse como cooperativas, en lugar de subversivas del sistema de dominación. Siendo el nivel de cooperación de los subordinados tanto activo como pasivo con su opresión que genera sistemas de jerarquía social grupal estables y rígidos. Por lo tanto, desde la SDT, la jerarquía social no se mantiene principalmente por el comportamiento opresivo de los dominantes, sino por el comportamiento deferente y obsequioso de los subordinados (Sidanius \& Pratto, 1999).

Sidanius y Pratto (1999) observan cuatro clases de comportamientos asimétricos vinculados al respeto de los subordinados a los grupos dominantes, el cual mantiene estable lajerarquía social. En primerlugar, el sesgo endogrupal asimétrico, en el cual los grupos dominantes tienen una tendencia a mostrar niveles más altos de favoritismo o parcialidad en el grupo que los subordinados, siendo de este modo más etnocéntricos. En segundo lugar, el favoritismo exogrupal que se define como la preferencia de alguno de los miembros de grupos desfavorecidos 
a favorecer a los grupos dominantes. En tercer lugar, la debilitación del self, que se presenta cuando los miembros del exogrupo realizan conductas autodestructivas que suelen estar relacionadas con el estereotipo del grupo. Finalmente, en cuarto lugar, la asimetría ideológica la cual se relaciona con las ideologías legitimadoras que incrementan la jerarquía, reforzando así en los grupos el apoyo-rechazo de la situación de desigualdad.

Estas formas de asimetría conductual dentro de la SDT dan cuenta de la cooperación de la opresión intergrupal en el marco de las jerarquías sociales. De esta manera, el sistema de jerarquía social basado en grupos no sólo es mantenida por la opresión activa de los dominantes o el cumplimiento pasivo de los subordinados, sino más bien por las actividades coordinadas y colaborativas de ambos (Pratto et al, 2006).

\section{Mitos legitimizantes}

De acuerdo con la SDT, la jerarquía social se apoya a su vez en los denominados mitos legitimizantes (Pratto et al, 1994) (Sidanius \& Pratto, 2004), los cuales se definen como actitudes, creencias, valores e ideologias aceptadas por la sociedad que legitiman la desigualdad. Los mitos legitimizantes se distinguen por dos características independientes: el tipo funcional y la potencia (Sidanius \& Pratto, 1999).

El tipo funcional da cuenta de sí un mito legitimizante en particular justifica la desigualdad social basada en el grupo o si justifica la igualdad social. Aquellos mitos que justifican la desigualdad social grupal son mitos legitimizantes promotores de la jerarquía, mientras que los mitos que justifican un mayor nivel de igualdad social son los mitos legitimizantes atenuantes de la jerarquía. Por ejemplo, el racismo, el sexismo, el nacionalismo o el clasismo suelen ser considerados mitos que 
acentúan la jerarquía social, promoviendo la desigualdad, mientras que los derechos del hombre, el universalismo o el multiculturalismo suelen considerarse ejemplos de mitos que mitigan la jerarquía social, promoviendo una mayor igualdad social (Levin et al, 2012).

La noción de potencia de un mito legitimizante, por otro lado, refiere al grado en que pueden promover, mantener o anular una determinada jerarquía social. Existen cuatro factores que influyen en este grado de poder: consensualidad, consistencia, certeza y fuerza mediadora.

La consensualidad se refiere a que mientras una creencia o ideologia es compartida en mayor medida por la sociedad, su impacto será mayor. De esta manera, si los prejuicios hacía un grupo son compartidos por todos los grupos el poder de este mito será más fuerte. Existen numerosos ejemplos de favoritismo exogrupal que muestran que las personas de color suelen apoyar en mayor medida a las de piel blanca, confirmando este proceso (Craig \& Richeson, 2014) (Jost \& Banaji, 1994) (Major, Blodorn \& Major Blascovich, 2018).

En segundo lugar, la consistencia supone que los mitos están fuertemente vinculados con características culturales que se encuentran arraigadas. Un ejemplo de ello puede ser el racismo hacía la gente de color, lo cual es consistente en la cultura occidental. En efecto, el color negro es por lo general asociado con cuestiones negativas -infierno, miedo, sucio-, a diferencia del color blanco-pureza, inocencia-(Williams, 1994). Por lo tanto, a medida que sea más consistente un mito con un factor cultural arraigado, mayor será su poder.

En tercer lugar, la certeza hace referencia a un grado alto de certeza o verdad moral, religiosa o científica. Un ejemplo de esto, es la creencia de una inferioridad genética de la gente de color, lo cual era muy extendido en el siglo XIX en Europa occidental. Esta creencia fue fuertemente 
basada en las teorias evolutivas de la época (Biddiss, 1970). Este racismo científico, aún inspira a ciertos investigadores (Herrnstein \& Murray, 1994) (Rushton, 1996). A mayor grado de certeza más poder por parte del mito legitimizante.

En cuarto lugar, la fuerza mediadora se remite al grado en que un mito legitimizante sirve como un enlace entre el deseo de establecer o mantener una jerarquía social grupal con el respaldo de las políticas sociales. Cuanto mayor es este grado, más fuerte es el poder del mito. Por ejemplo, las personas que apoyan la ética laboral protestante, suelen ser los más oposicionistas a la ayuda para los pobres y menos afortunados. Según SDT, la razón por la que los individuos apoyan a la ética protestante de trabajo, se debe a que es un medio accesible y socialmente aceptado de justificar la desigualdad (Sidanius \& Pratto, 1999).

Por otro lado, los mitos legitimizantes se sirven de ciertas ideologías que se encuentran fuertemente asociado a la SDO (Lee et al, 2011). Las personas en ocasiones utilizan ideologías para justificar su comportamiento, guiar sus propias acciones y determinar qué comportamiento en otros individuos sienten que está justificado. Frente a un cambio social, las ideologías suelen ser importantes, oponiéndose o acogiendo a las mismas ideologías y para apoyar $u$ oponerse a diversos tipos de cambio social. Pratto et al (1994), sugieren que hay una serie de ideologias sociales muy útiles para los mitos legitimizantes.

En primer lugar, mencionan el prejuicio étnico, que es entendido como cualquier ideología que describa a los grupos como desiguales y tenga implicaciones políticas (Pratto et al, 1994) sin importar si la base del racismo es la equidad (Kluegel \& Smith, 1986), las teorias de inferioridad racial genética o bíblica, el racismo simbólico (Sears, 1988) o la patología familiar (Moynihan, 1967). 
En segundo lugar, otra ideología muy frecuente es el nacionalismo que es un tipo de prejuicio en grupo que puede ocurrir en los estados nacionales.

Kosterman y Feshbach (1989) sugirieron que los sentimientos de los países -patriotismo- se puede distinguir del prejuicio comparativo, es decir, que el país de uno es mejor que otros países -nacionalismo-, y como tal debe dominar a otros países -chovinismo-. No obstante, los tres reflejan un sesgo actitudinal a favor de lo nacional dentro del grupo.

En tercer lugar, el elitismo cultural que remite a que todas las sociedades comparten la idea de que una de las características definitorias de aquellos que pertenecen a su sociedad es que son "cultos". Una ideología elitista construida sobre la distinción culto - no culto, postula que la clase de élite tiene una "cultura" no compartida por la clase media y la clase trabajadora y por lo tanto es más merecedora de mejores cosas.

En cuarto lugar, el sexismo que se refiere a la ideología sexista y varia ampliamente con la religión, la historia culturaly la tecnología. Se piensa a este mito como la medida en que las personas creen que los hombres y las mujeres son "naturalmente" diferentes, debido a lo cual deberian tener diferentes trabajos y roles tanto fuera como dentro del hogar (Benson \& Vincent, 1980) (Rombough \& Ventimiglia, 1981). Y también en qué medida las personas creen que se puede culpar a las mujeres en lugar de a los hombres por los avances sexuales no deseados, como la violación y el acoso sexual (Burt, 1980).

En quinto lugar, el conservadurismo político-económico, el cual se asocia con el apoyo para el capitalismo contra el socialismo (Eysenck, 1971). Dado que el capitalismo implica que algunas personas y empresas deberian prosperar, mientras que aquellos que son menos competitivos 
no deberian hacerlo. Dividir a las personas en grupos que "merecen" un tratamiento diferente, siendo el conservador aquel que muestra una preferencia por las relaciones sociales jerárquicas.

En sexto lugar la nobleza obliga que es una ideología que atenúa la jerarquía que existe en muchas culturas. Dado que aquellos con más recursos deberian compartirlos con aquellos que tienen menos recursos.

En séptimo lugar, la meritocracia que es una ideología que mejora la jerarquía, ya que se piensa que la riqueza y los otros valores sociales están distribuidos adecuadamente, basados en el mérito de los destinatarios. La ética del trabajo protestante y la creencia en un mundo justo son ejemplos de ideologías meritocráticas. En los Estados Unidos, las atribuciones a la pobreza por pereza o por algunas otras faltas inherentes en los pobres se basan en la idea que la igualdad de oportunidades está disponible para todos (Kluegel \& Smith, 1986).

En octavo lugar, las actitudes de política social se refieren a que los individuos orientados a la dominación social favorecerán las prácticas sociales que mantienen o exacerban la desigualdad entre los grupos y se opondrán a las prácticas sociales que reducen la desigualdad grupal.

En noveno lugar, el bienestar social, los derechos civiles y las políticas ambientales, entendida como la oposición a políticas sociales que reducirian la desigualdad entre las personas que habitan una nación y extranjeros o inmigrantes, ricos y de clase media o pobres, hombres y mujeres, grupos étnicos, heterosexuales y homosexuales, y los humanos frente a otras especies.

En décimo lugar, la politica militar que se piensa como la expresión positiva de los programas y acciones militares, siendo que el ejército es un símbolo del nacionalismoy puede ser uno de los principales medios de dominación de una nación por sobre otras. 
Finalmente, las politicas punitivas, a pesar de la creencia de que se promulga la igualdad ante la ley, existen investigaciones de cómo el punitivismo no promulga dicha igualdad, como por ejemplo en el sistema de justicia penal de EE. UU. en el cual, se muestra sesgos de clase y etnia en todos los niveles, desde el arresto hasta la negociación de culpabilidad y la sentencia (Bienen, Alan, Denno, Allison \& Mills, 1988) (General Accounting Office, 1990) (Nickerson, Mayo \& Smith, 1986) (Radelet \& Pierce, 1985) (Reiman, 1990) (Sidanius, 1988).

Todas estas ideologías refuerzan los mitos legitimizantes aumentando una orientación a la dominancia social por parte de los individuos y contribuyendo a aumentar las jerarquías o a disminuirlas (Pratto et al, 2006).

\section{Orientación a la Dominancia Social: "mejorar la jerarquia" vs "atenuación de jerarquia"}

De acuerdo con SDO, las dinámicas intergrupales se ven mayormente influenciadas por las posiciones del individuo en la sociedad (Sidanius \& Pratto, 1999). Si bien, los conceptos de estatus y poder son distintos, estos se ven a menudo estrechamente relacionados, dado que las personas o grupos en la parte superior de la estructura social suelen tener una posición más alta, teniendo por lo general mayor poder. Esta habilidad posibilita controlar los resultados de otros (Goodwin, Operario $\&$ Fiske, 1998) (Lemieux \& Pratto, 2003) (Richeson \& Ambady, 2003). Distintos investigadores (Fiske, 1993) (Goodwin, Gubin, Fiske \& Yzerbyt, 2000) (Pratto \& Pitpitan, 2008) (Rodriguez-Baillon, Moya \& Yzerbyt, 2000), muestran que los individuos en una situación de poder expresan más estereotipos y juicios negativos. Esto les permite a los individuos, mantener una posición de poder que en algunos casos genera actitudes 
negativas y comportamientos hostiles hacia grupos sociales subordinados (Georgesen \& Harris, 1998) (Kipnis, 1972). No obstante, en las investigaciones sobre estos efectos del poder, hay que tener en cuenta la influencia del contexto normativo basado en la distinción realizada por Sidanius y Pratto (1999) entre: entornos de "mejorar la jerarquia" -HEvs entornos de "atenuación de jerarquía" -HA-.

Estos entornos están formados por organizaciones o instituciones que favorecen o reducen las desigualdades sociales y las relaciones de dominancia. De este modo, cada tipo de entorno es manejado por normas específicas, constituyendo así entornos normativos distintos. El sistema de justicia penal, el ejército, la policía y algunas empresas grandes, son ejemplos de entornos normativos que se caracteriza por ser desiguales, ya que facilitan el reparto de recursos sociales negativos en relación a los grupos subordinados (Ginet, Guimond \& Greffeuille, 2006) (Guimond, 2000) (Poteat, Espelage \& Green, 2007) (van Laar, Sidanius, Rabinowitz \& Sinclair, 1999). Por otro lado, los servicios sociales, asociaciones humanitarias, organizaciones para la defensa de los derechos humanos y algunos sindicatos, son entidades que representan entornos normativos pro-igualitarios (Sidanius \& Pratto, 1999) (Pratto et al, 2006). Estas entidades, tienen como función principal, reducir las desigualdades sociales buscando por ejemplo establecer una redistribución equitativa de los recursos.

Diversos estudios han dado cuenta de que las personas que pertenecen a $\mathrm{HE}$, tienden a ser entornos normativos más desiguales e intolerantes con los grupos desfavorecidos que con los miembros de alta disponibilidad (Dambrun, Guimond \& Duarte, 2002) (Gatto, Dambrun, Kerbrat \& De Oliveira, 2010) (Sidanius, Liu, Pratto \& Shaw, 1994) (Poteat et al, 2007) (van Laar et al, 1999). Los autores Guimond, Dambrun, Michinov y Duarte (2003) explican en relación a los entornos normativos 
de HE y HA, que la socialización en un contexto normativo dado, conlleva a los miembros del mismo a la adquisición de un conjunto de normas, valores, comportamientos y actitudes, apropiadas para el entorno.

\section{Otras implicaciones estructurales de SDT}

Los mecanismos de la SDT, no solo hacen que las jerarquías sociales basadas en grupos sean estables y ubicuas, sino que a la vez proporcionan otras características. Tres de estas implicaciones estructurales son: el aumento de la desproporcionalidad, consensualidad jerárquica y la resistencia.

El aumento de la desproporcionalidad hace referencia a lo que Putnam (1976) denominó, la ley de desproporción creciente. Esta ley, estipula que a medida que una posición ejerza mayor influencia politica, ya sea ministro, primer ministro, presidente, entre otros, es más probable que la constitución del cargo sea ocupado por miembros de los grupos dominantes. Además, esta ley opera sobre las tres formas de estratificación grupal-edad, género, grupos arbitrarios-. Por último, para Putnam (1976) esta desproporcionalidad creciente, no está restringida a culturas o naciones particulares, sino que se encuentra de manera transcultural en países como: Estados Unidos, Rusia o Túnez. En segundo lugar, la consensualidad jerárquica se refiere al consenso entre los grupos dominantes y subordinados, en relación a la estructura del sistema social y el estado de los grupos que lo componen (Sidanius \& Pratto, 1999).

Finalmente, la resistencia da cuenta que si bien las jerarquías de grupos sociales, tienden a ser estables en el transcurso del tiempo, esta estabilidad no es absoluta. Dentro de cualquier sistema social, el grado de jerarquía social no solo varía a lo largo del tiempo, sino que también 
varía menos en el sistema de conjunto arbitrario y existen ocasiones escasas en las cuales una determinada jerarquía social grupal será derrocada. Se estipula que ha habido al menos siete eventos de revoluciones sociales en los últimos 300 años (Sidanius \& Pratto, 1999): la revolución francesa de 1789, la revolución mexicana de 1910, la revolución rusa de 1917, la revolución china de 1949, la revolución vietnamita (1954-1975), la revolución cubana de 1959, y la revolución sandinista de 1979.

Sin embargo, por más que ha habido intentos de transformación social igualitaria, es de destacarse que no existe un solo caso en el que una transformación igualitaria haya tenido un éxito real. Esto se debe a que incluso en los casos en que el antiguo régimen fue derrocado, emerge pronto un nuevo conjunto que restablece el orden ocupando así el lugar vacío. De esta manera, por más que un determinado sistema de estratificación de conjunto pueda colapsar o ser derrocado, el fenómeno de estratificación de conjuntos parece ser flexible.

\section{Evaluación de la Orientación a la Dominancia Social}

La teoría de la dominancia social estipula que los fundamentos que mantienen la desigualdad social son reforzados mediante un mecanismo psicológico llamado SDO, el cual puede ser estudiado en el ámbito individual (Pratto et al, 1994).

A fin de explicar los mecanismos que rigen la producción y el mantenimiento de las jerarquias sociales, Pratto et al (1994) desarrollaron la Escala de SDO, la cual es una herramienta que permite concebir una medición en relación a la predisposición que tienen los individuos hacia el mantenimiento de las relaciones intergrupales de carácter jerárquico y no igualitario. La SDO es considerada una 
orientación actitudinal general hacia las relaciones intergrupales, que refleja si generalmente se prefieren relaciones igualitarias o jerárquicas.

Luego de la construcción definitiva de la escala SDO (Pratto et al, 1994), la misma se demostró como un fuerte predictor de diferentes tipos de prejuicio, como pueden ser el prejuicio étnico (Bates \& Heaven, 2001) (Duriez \& van Hiel, 2002), el racismo clásico (Sidanius, Pratto \& Bobo, 1996) o el sexismo (Pratto et al, 1994) (Sidanius \& Pratto, 1999, 2004) (Sidanius et al, 2004). Además, este constructo se encuentra asociado a los valores, el autoposicionamien to ideológico-politico (Brussino, Rabbia, Imhoff \& Garcia, 2011) (Sibley, Osborne \& Duckitt, 2012) (Zubieta, Delfino \& Fernández, 2007), el conservadurismo político, el nacionalismo, el patriotismo (Pratto et al, 1997), la oposición a políticas medioambientales, los derechos civiles, el estado de bienestar (Sidanius et al, 2004) y las políticas económicas conservadoras (Pratto et al, 1994).

Uno de los resultados más importantes que trajo aparejado las diversas investigaciones con la escala SDO, es el denominado invarianza de género, el cual determina que los varones obtienen mayores puntuaciones en SDO que las mujeres en las diferentes culturas en las cuales se realizó la investigación.

\section{Discusión}

La SDT ha atraído el interés reciente de los psicólogos sociales, ya que tiene profundas implicaciones para la comprensión de los conflictos intergrupales, particularmente en relación con factores como los prejuicios y la discriminación, así como la resolución de los conflictos. Sin embargo, quizás el punto más importante de la teoría sea que es una invariante psicológica en la naturalización de la desigualdad. En este 
sentido, este trabajo tiene como objetivo presentar una revisión exhaustiva de la SDT.

Entendemos que las distintas perspectivas para el estudio del fenómeno de la desigualdad han sido generalmente interpretadas como sustitutivas y antagónicas, producto de la hegemonía en las perspectivas de análisis top down. La irrupción de perspectivas psicológicas bottom up ha permitido desarrollar otra mirada diferente para la explicación de este fenómeno. Si bien, las primeras han criticado tradicionalmente a las segundas por el hecho de que la asunción de una explicación psicológica posibilita la apropiación de una visión del mundo que termine legitimando y aceptando las diferencias sociales existentes, la SDT se distancia de estas lecturas psicologicista en varios aspectos y entendemos que no colabora en la invisibilización y reificación de distintas filosofias de la desigualdad social. Lejos de desresponsabilizar a las clases dominantes, y focalizar la culpa en una responsabilidad cognitiva individual, la SDT ha demostrado en su corpus teórico herramientas conceptuales como los mitos legitimizantes, la discriminación individual e institucional, y la asimetría conductual, para lograr una buena sintesis entre elementos psicológicos y sociológicos realizando una sintesis dialéctica superadora.

Finalmente, entendemos que la sociologización o psicologización del fenómeno, no alcanzan y que es necesario un abordaje interdisciplinario con teorias superadoras como la SDT, ya que por un lado se encuentra la influencia de los grupos dominantes en la ideología que se difunde entre los grupos dominados - top down-, mientras que por el otro lado existen diferencias en distintas variables psicológicas -bottom up- que afecta la adhesión a la ideología del individuo. 


\section{Referencias}

Adorno, T., Frenkel-Brunswik, E., Levinson, D., \& Sanford, R. (1950). The authoritarian Personality. New York: Harper.

Alves, J. A. (2019). Setting the Tone: Micro/Macro Racial Aggression, Antiblackness and the Outlining of a Trans-National Research Agenda on Community Response s to State Terror. NCID Currents, 1(1).

Amnesty International USA, \& United States of America. (1999). Killing With Prejudice: Race and the Death Penalty.

Angus, J., \& Crichlow, V. (2018). A Race and Power Perspective on Police Brutality in America. FAURJ, 8.

Bates, C., \& Heaven, P. (2001). Attitudes to women in socie ty: the role of social dominance orientation and social values. Journal of Community \& Applied Social Psychology, 11, 43-49. https: / / doi.org/10.1002/casp.589

Benson, P. L., \& Vincent, S. (1980). Development and Validation of the Sexist Attitudes Toward Women Scale (SATWS). Psychology of Women Quarterly, 5(2), 276-291. https://doi.org/10.1111/j.1471-6402.1980.tb00962.x

Bernard, P. (1993). L'immigration. Le Monde-Editions.

Biddiss, M. D. (1970). Father of racist ideology: The social and political thought of Count Gobineau. London: Weidenfeld \& Nicolson.

Bienen, L. B., Weiner, N. A., Denno, D. W., \& Allison, P. D. (1988). The reimposition of capital punishment in New Jersey: The role of prosecutorial discretion. Rutgers L. Rev., $41,27$.

Bonanno, G. A., Jost, J. T. (2006). Conservative shift among high-exposure survivors of the Se ptember 11th terrorist attacks. Basic and Applied Social Psychology, 28, 311-323. https://doi.org/10.1207/s 15324834basp2804_4

Brewer, M. B. (2001). Ingroup identification and in tergroupconflict. Social identity, intergroup conflict, and conflict reduction, 3, 17-41.

Brussino, S. A., Rabbia, H. H., Imhoff, D., \& García, A. P. P. (2011). Dimensión operativa de la ideología política en ciudadanos de Córdoba. Psicología Política, (43), 85-106. 
Burt, M. R. (1980). Cultural myths and supports for rape. Journal of Personality and Social Psychology, 38(2), 217-230. http://dx.doi.org/10.1037/0022-3514.38.2.217

Cárdenas, M., Mesa, P., Lagues, K., \& Yañez, S. (2010). Adaptación y validación de la Escala de Orientación a la Dominancia Social (SDO) en una mue stra chilena. Universitas Psychologica, 9, 161-168.

CNCDH (Commission Nationale Consultative des Droits de l'Homme). (1999). La lutte contre le racisme et la xénophobie, Paris: La documentation Française.

Converse, P. E. (1964). The nature of belief systems in mass publics. Ideology and discontent. Ideology and Discontent, 206-261.

Craig, M. A., \& Richeson, J. A. (2014). On the precipice of a "majority-minority" America: Perceived status threat from the racial de mographic shift affects white Americans' political ide ology. Psychological Science, 25, 1189-1197. https: / /doi.org/ 10.1177/0956797614527113

Dambrun, M., Guimond, S., \& Duarte, S. (2002). The impact of hierarchy-enhancing vs. attenuating academic major on stereotyping: The mediating role of perceived social norms. Current Re search in Social Psychology, 7, 114-136.

Dovidio, J. F., Hewstone, M., Glick, P., \& Esses, V. M. (2010). Prejudice, Stereotyping and Discrimination: Theoretical and Empirical Overview. In J. F. Dovidio, M. Hewstone, P. Glick, \& V. M. Esses (Eds.), The SAGE Handbook of Prejudice, Stereotyping and Discrimination (pp. 3-28). London: Sage Publications Ltd. https: / /doi.org/ 10.4135/9781446200919.n1

Duriez, B., \& van Hiel, A. (2002). The march of modern fascism. A comparison of social dominance orientation and authoritarianism. Personality and Individual Diffe rences, 32(7), 1199-1213. http://dx.doi.org/10.1016/S0191-8869(01)00086-1

Engels, F. (1884/1902). The origin of the family, private property, and the state (E. Untermann, Trans.). Chicago: E. H. Kerr.

Etchezahar, E., Prado-Gascó, V., Jaume, L., \& Brussino, S. (2014). Validación argentina de la Escala de Orientación a la Dominancia Social. Revista Latinoamericana de Psicología, $46(1), 35-43$.

Eysenck, H. J. (1971). Social attitudes and social class. British Journal of Social \& Clinical Psychology, 10(3), 201-212. http://dx.doi.org/10.1111/j.2044-8260.1971.tb00738.x

Feagin, J. R., \& Feagin, C. B. (1978). Discrimination American style: Institutional racism and sexism. Englewood Cliffs, N.J: Prentice-Hall. 
Ferguson, C. J., \& Dyck, D. (2012). Paradigm change in aggression re search: The time has come to retire the General Aggression Model. Aggression and Violent Behavior, 17(3), 220-228. http://dx.doi.org/10.1016/j.avb.2012.02.007

Fiske, S. T. (1993). Controlling other people: The impact on stereotyping. American Psychologist, 48, 621-628.

Gatto, J., Dambrun, M., Kerbrat, C., \& De Oliveira, P. (2010). Prejudice in the police: On the processe s underlying the effects of selection and group socialization. European Journal of Social Psychology, 40, 252-269.

General Accounting Office. (1990). Death penalty sentencing: Researchindicates pattern of racial disparities. United States General Ac-counting Office, Report to Senate and House Committees on the Ju-diciary (GAO/GGD-90-57). Washington, DC: Government PrintingOffice.

Georgesen, J. C., \& Harris, M. J. (1998). Why's my boss always holding me down? A metaanalysis of power effects on performance evaluation. Personality and Social Psychology Review, 2, 184-195.

Ginet, M., Guimond, S., \& Greffeuille, C. (2006). Human justice or injustice? The jury system in France. In M. F. Kaplan \& A. M. Martin (Eds.), Understanding world jury systems through psychological re search (pp. 147-164). New York, NY: Psychology Press.

Goodwin, S. A., Gubin, A., Fiske., S. T., \& Yzerbyt, V. Y. (2000). Power can bias impression processes: Stereotyping subordinates by default and by design. Group processes and intergrouprelations, 3, 227-256.

Goodwin, S. A., Operario, D., \& Fiske, S. T. (1998). Situational power and interpersonal dominance facilitate bias and inequality. Journal of Social Issues, 54, 677-698.

Guimond, S. (2000). Group socialization and prejudice: The social transmission of intergroupattitudes and beliefs. European Journal of Social Psychology, 30, 335-354.

Guimond, S., Dambrun, M., Michinov, N., \& Duarte, S. (2003). Does social dominance generate prejudice? Inte grating individual and contextual de terminants of intergroup cognitions. Journal of Personality and Social Psychology, 84, 697-721.

Herrnstein, R. J., \& Murray, C. A. (1994). The bell curve: Intelligence and class structure in American life. New York: Free Press.

Jost, J. T. (2006). The end of the end of ideology. American Psychologist, 61(7), 651-670. https://doi.org/10.1037/0003-066X.61.7.651 
Jost, J. T., \& Banaji, M. R. (1994). The role of stereotyping in system-justification and the production of false-consciousness. British Journal of Social Psychology, 33, 1-27.

Jost, J. T., Glaser, J., Kruglanski, A. W., Sulloway, F. J. (2003). Political conservatism as motivated social cognition. Psychological Bulletin, 129, 339.

https: / /doi.org/ 10.1037/0033-2909.129.3.339

Jost, J., \& Thompson, E. (2000). Group-based dominance and opposition to equality as independent predictors of self-esteem, ethnocentrism, and social policy attitudes among African Americans and Europe an Americans. Journal of Experimental Social Psychology, 36, 209-232. https://doi.org/ 10.1006/jesp.1999.1403

Kipnis, D. (1972). Does power corrupt? Journal of Personality and Social Psychology, 24, $33-41$.

Kosterman, R., \& Feshbach, S. (1989). Toward a Measure of Patriotic and Nationalistic Attitudes. Political Psychology, 10(2), 257-274. http: / dx.doi.org/ 10.2307/3791647

Klue gel, J. R., \& Smith, E. R. (1986). Beliefs about inequality: Americans' views of what is and what ought to be. New York: Aldine De Gruyter.

Lange, A. (1996). Invandrare om diskriminering II. En enka"t- och intervjuunderso"kning om etnisk diskriminering $\mathrm{pa}^{\circ}$ uppdrag av Diskrimineringsombudsmannen (DO) (Immigrants on discrimination II), CEIFO, Stocholm University.

Lee, I., Pratto, F., \& Johnson, B. T. (2011). Intergroup consensus/disagreement in support of group-based hierarchy: An examination of socio-structural and psycho-cultural factors. Psychological Bulletin, 137, 1029-1064.

Lemieux, A. F., \& Pratto, F. (2003). Poverty and prejudice. In S. Carr \& T. Sloan (Eds.), Poverty and psychology: From global perspective to local practice (pp. 147-161). New York, NY: Kluwer.

Levin, S., Matthews, M., Guimond, S., Sidanius, J., Pratto, F., Kteily, N. \& Dover, T. (2012). Assimilation, multiculturalism, and colorblindness: Me diated and moderated relationships between social dominance orientation and prejudice. Journal of Experimental Social Psychology, 48(1), 207-212. https: / /doi.org/ 10.1016/j.jesp.2011.06.019

Major, B., Blodorn, A., \& Major Blascovich, G. (2018). The threat of increasing diversity: Why many White Americans support Trump in the 2016 presidential election. Group Processes \& Intergroup Relations, 21(6), 931-940.

https:/ / doi.org/ 10.1177/1368430216677304 
Martín-Baró, Ignacio. (1983/2004). Acción e ideología. Psicología social de sde Centroamérica. El Salvador: UCA Editores.

Marx, K., \& Engels, F. (1846/1970). The German ideology. New York: International Publishers.

Moore, S. E., Robinson, M. A., Clayton, D. M., Adedoyin, A. C., Boamah, D. A., Kyere, E., \& Harmon, D. K. (2018). A Critical Race Perspective of Police Shooting of Unarmed Black Males in the United States: Implications for Social Work. Urban Social Work, 2(1), 3347.

Moynihan, D. P. (1967). The Negro family: The case for national action. In L. Rainwater \& W. L. Rainwater (Eds.), The Moynihan Report and the politics of controversy (pp. 41-64). Cambridge, MA: MIT Press.

Myers, D. G., \& Lamarche, L. (1992). Psychologie sociale. Montréal: McGraw-Hill.

Nickerson, S., Mayo, C., \& Smith, A. (1986). Racism in the courtroom. In J. F. Dovidio \& S. L. Gaertner (Eds.), Prejudice, discrimination, and racism (pp. 255-278). San Diego, CA, US: Academic Press.

Poteat, V. P., Espelage, D. L., \& Green, H. D. (2007). The socialization of dominance: Peer group contextual effects on homophobic and dominance attitudes. Journal of Personality and Social Psychology, 92, 1040-1050.

Pratto, F. (2015). On power and empowerment. British Journal of Social Psychology, 55, 1 20. https://doi.org/10.1111/bjso. 12135

Pratto, F., Liu, J. H., Levin, S., Sidanius, J., Shih, M., Bachrach, H., \& Hegarty, P. (2000). Social dominance orie ntation and the legitimization of inequality across cultures. Journal of Cross-Cultural Psychology, 31(3), 369-409. http: / /dx.doi.org/ 10.1177/0022022100031003005

Pratto, F., \& Pitpitan, E. (2008). Ethnocentrism and sexism: How stereotypes legitimize six types of power. Social and Personality Psychology Compass, 2, 2159-2176.

Pratto, F., Sidanius, J., \& Levin, S. (2006). Social Dominance The ory and the dynamics of intergroup relations: Taking stock and looking forward. European Review of Social Psychology, 17, 271-320. https://doi.org/10.1080/10463280601055772

Pratto, F., Sidanius, J., Stallworth, L. M., \& Malle, B. F. (1994). Social Dominance Orientation: A personality variable predicting social and political attitudes. Journal of Personality and Social Psychology, 67, 741-763. https://doi.org/10.1037/00223514.67.4.741 
Pratto, F., Stallworth, L. M., Sidanius, J., \& Siers, B. (1997). The gender gap in occupational role attainment: A social dominance approach. Journal of Personality and Social Psychology, 72(1), 37-53. http:/ / dx.doi.org/ 10.1037/0022-3514.72.1.37

Putnam, R. D. (1976). The comparative study of political elites. Englewood Cliffs, NJ: Prentice-Hall.

Radelet, M. L., \& Pierce, G. L. (1985). Race and prosecutorial discretion in homicide cases. Law \& Socie ty Review, 19(4), 587-621. http: / dx.doi.org/10.2307/3053422

Reiman, J. (1990). The rich get richer and the poor get prison: Ideology,class, and criminal justice. New York: Macmillan.

Richeson, J. A., \& Ambady, N. (2003). Effects of situational power on automatic racial prejudice. Journal of Experimental Social Psychology, 39, 177-183.

Rodriguez-Baillon, R., Moya, M., \& Yzerbyt, V. Y. (2000). Why do superiors attend to negative stereotypic information about their subordinates? Effects of power le gitimacy on social perce ption. European Journal of Social Psychology, 30, 651-671.

Rombough, S., \& Ventimiglia, J. C. (1981). Sexism: A tri-dimensional phenomenon. Sex Roles: A Journal of Research, 7(7), 747-755. http: / /dx.doi.org/ 10.1007/BF00290042

Rushton, J. P. (1996). Race diffe rences in brain size. American Psychologist, 51, 556.

Sears, D. O. (1988). Symbolic racism. In P. A. Katz \& D. A. Taylor (Eds.), Eliminating racism: Profiles in controversy (pp. 53-84). New York: Plenum.

Sherif, M., Harvey, O. J., White, B. J., Hood, W. R., \& Sherif, C. W. (1961). Intergroup conflict and cooperation: The Robber's Cave experiment. Norman: University of Oklahoma Press.

Sibley, C. G., Osborne, D., \& Duckitt, J. (2012). Personality and political orientation: me taanalysis and test of a threat constraint model. Journal of Research in Personality, 46, 664- 677. https://doi.org/10.1016/j.jrp.2012.08.002

Sidanius, J. (1988). Race and Sentence Severity: The Case of American Justice. Journal of Black Studies, 18(3), 273-281. https://doi.org/10.1177/002193478801800302

Sidanius, J. (1993). The psychology of group conflict and the dynamics of oppre ssion: A social dominance perspective. In S.Iyengar, \& W.J. McGuire (Eds.), Explorations in political psychology (pp. 183-219). Durham, NC: Duke University Press. 
Sidanius, J., Cotterill, S., She ehy-Skeffington, J., Kteily, N., \& Carvacho, H. (2017). Social dominance theory: Explorations in the psychology of oppression. In C. G. Sibley \& F. K. Barlow (Eds.), The Cambridge handbook of the psychology of prejudice (p. 149-187). Cambridge University Press. https://doi.org/10.1017/9781316161579.008

Sidanius, J., Levin, S., Liu, J., \& Pratto, F. (2000). Social dominance orientation, anti egalitarianism and the political psychology of gender: An extension and cross-cultural re plication. Europe an Journal of Social Psychology, 30(1), 41-67. http: / / dx.doi.org/ 10.1002/(SICI) 1099-0992(200001/02)30:1<41::AIDEJSP976>3.0.CO;2-O

Sidanius, J., Liu, J., Pratto, F., \& Shaw, J. (1994). Social dominance orientation, hierarchyattenuators and hierarch-yenhancers: Social the ory and the criminal justice system. Journal of Applied Social Psychology, 24, 338-366.

Sidanius, J., \& Pratto, F. (1999). Social dominance: An intergroup theory of social hierarchy and oppression. Cambridge: Cambridge University Press. https:// doi.org/10.1017/CBO9781139175043

Sidanius, J., \& Pratto, F. (2004). Social dominance theory: A new synthesis. In J. T. Jost \& J. Sidanius (Eds.), Key readings in social psychology. Political psychology: Key readings (p. 315-332). Psychology Press. https:// doi.org/10.4324/9780203505984-18

Sidanius, J., Pratto, F., \& Bobo, L. (1994). Social dominance orientation and the political psychology of gender: A case of invariance. Journal of Personality and Social Psychology, 67, 998-1100. https://doi.org/10.1037/0022-3514.67.6.998

Sidanius, J. Pratto, F., \& Bobo, L. (1996). Racism, conservatism, affirmative action and intellectual sophistication: A matter of principled conservatism or group dominance? Journal of Personality and Social Psychology, 70, 476-490.

Sidanius, J., Pratto, F., \& Brief, D. (1995). Group dominance and the political psychology of gender: A cross-cultural comparison. Political Psychology, 16, 381-396. http: / /dx.doi.org/ 10.2307/3791836

Sidanius, J., Pratto, F., van Laar, C., \& Levin, S. (2004). The Social Dominance Theory: Its age nda and method. Political Psychology, 25:845-800. https://doi.org/10.1111/j.14679221.2004.00401.x

Silván-Ferrero, M. P., \& Bustillos, A. (2007). Adaptación de la Escala de Orientación a la Dominancia Social al castellano: validación de la Dominancia Grupal y la Oposición a la Igualdad como factores subyacentes. Revista Psicologia Social, 22, 3-15.

Tonry, M. (2010). The social, psychological, and political causes of racial disparities in the American criminal justice system. Crime and justice, 39(1), 273-312. https://doi.org/10.1086/653045 
van Laar, C., Sidanius, J., Rabinowitz, J., \& Sinclair, S. (1999). The three r's of academic achievement: Reading, 'riting, and racism. Personality and Social Psychology Bulletin, 25, 139-151.

Williams Jr, R. M. (1994). The sociology of ethnic conflicts: comparative international perspectives. Annual review of sociology, 20(1), 49-79.

Zakrisson, I. (2008). Gender Differences in Social Dominance Orientation: Gender Invariance May Be Situation Invariance. Sex Roles 59, 254. https:/ / doi.org/ 10.1007/s 11199-008-9445-Z

Zubieta, E., Delfino, G., \& Fernández, O. (2007). Dominancia social, valore s y posicionamiento ideológico en jóvenes universitarios. Psicode bate, 8, 151-170. 